\title{
Téoros
}

Revue de recherche en tourisme

\section{Le contrôle à distance}

\section{La gestion de la sous-traitance au Kirghizstan par les agences françaises de tourisme d'aventure}

\section{Julie Hallé et Eric Passavant}

Volume 31, numéro 1, 2012

URI : https://id.erudit.org/iderudit/1020713ar

DOI : https://doi.org/10.7202/1020713ar

Aller au sommaire du numéro

\section{Éditeur(s)}

Université du Québec à Montréal

ISSN

0712-8657 (imprimé)

1923-2705 (numérique)

Découvrir la revue

Citer cet article

Hallé, J. \& Passavant, E. (2012). Le contrôle à distance : la gestion de la sous-traitance au Kirghizstan par les agences françaises de tourisme d'aventure. Téoros, 31(1), 103-114. https://doi.org/10.7202/1020713ar

\section{Résumé de l'article}

Les contraintes liées à la concurrence et aux fluctuations du tourisme international ont conduit les agences françaises de tourisme d'aventure à modifier leurs conditions de production. Aujourd'hui, elles sous-traitent à des agences locales une grande partie de la fabrication et de l'accompagnement des voyages qu'elles vendent. L'objectif de l'article est d'interroger les enjeux de cette évolution et d'identifier les stratégies mises en oeuvre par les agences pour maintenir la valeur distinctive de leurs voyages et répondre aux attentes de la clientèle française. Ce texte repose sur une enquête de terrain réalisée auprès des responsables d'agences françaises mais aussi de leurs correspondants locaux. Plusieurs séjours au Kirghizstan entre 2003 et 2006 nous ont permis d'étudier le fonctionnement de 28 agences locales et de connaître les trajectoires de leurs fondateurs. Il apparaît ainsi qu'une relation de confiance se nouera d'autant plus facilement entre une agence française et son correspondant étranger que celui-ci est un expatrié, qui s'est imposé localement en développant un réseau local, notamment à partir de stratégies matrimoniales.
Ce document est protégé par la loi sur le droit d'auteur. L'utilisation des services d'Érudit (y compris la reproduction) est assujettie à sa politique d'utilisation que vous pouvez consulter en ligne.

https://apropos.erudit.org/fr/usagers/politique-dutilisation/ 


\section{Le contrôle à distance La gestion de la sous-traitance au Kirghizstan par les agences françaises de tourisme d'aventure}

\author{
Julie HALLÉ \\ Chercheure associée \\ Laboratoire SENS (Sport et ENvironnement Social) \\ Université Joseph Fourier, Grenoble (France) \\ julie.halle@voila.fr
}

\author{
Eric PASSAVANT \\ Maître de Conférences \\ Centre Universitaire de Recherche \\ sur l'Action Publique et le Politique \\ Université de Picardie Jules Verne, Amiens (France) \\ eric.passavant@u-picardie.fr
}

RÉSUMÉ: Les contraintes liées à la concurrence et aux fluctuations du tourisme international ont conduit les agences françaises de tourisme d'aventure à modifier leurs conditions de production. Aujourd'hui, elles sous-traitent à des agences locales une grande partie de la fabrication et de l'accompagnement des voyages qu'elles vendent. L'objectif de l'article est d'interroger les enjeux de cette évolution et d'identifier les stratégies mises en œuvre par les agences pour maintenir la valeur distinctive de leurs voyages et répondre aux attentes de la clientèle française. Ce texte repose sur une enquête de terrain réalisée auprès des responsables d'agences françaises mais aussi de leurs correspondants locaux. Plusieurs séjours au Kirghizstan entre 2003 et 2006 nous ont permis d'étudier le fonctionnement de 28 agences locales et de connaître les trajectoires de leurs fondateurs. II apparaît ainsi qu'une relation de confiance se nouera d'autant plus facilement entre une agence française et son correspondant étranger que celui-ci est un expatrié, qui s'est imposé localement en développant un réseau local, notamment à partir de stratégies matrimoniales.

Mots-clés: Voyagiste, forfait, sous-traitance, aventure, Kirghizstan.

L'obsession du nombre, la recherche de la rareté, l'investissement «d'espaces libres et sans maître» sont des composantes essentielles du rapport au monde des membres de la bourgeoisie (Boltanski, 1976 : 102). À la fin des années 1970, l'accroissement des taux de départ en vacances des catégories moyennes et populaires façonne dans les catégories supérieures un sentiment d'envahissement par des masses de touristes (Réau, 2011 : 97). De nouvelles agences de voyage apparaissent et prennent en charge ce désir de distinction sociale (Bourdieu, 1979) en mobilisant la thématique de l'aventure contre celle du tourisme, accablé de tous les défauts depuis la fin du XIX ${ }^{e}$ siècle (Urbain, 1991 : 12). Elles inventent le voyage aventure, une "aventure sans risque» (Le Breton, 1996 : 127). Il s'agit d'un circuit organisé pour des groupes de taille réduite, avec un forfait tout compris, dans des régions isolées, peu connues et peu fréquentées. Le voyage aventure suppose l'adoption d'une éthique de l'effort et de l'inconfort (Tinard, $1992: 10)$ : les déplacements s'effectuent souvent à pied et les conditions de vie sont rudimentaires. Cette éthique s'inscrit dans une recherche d'authenticité, c'est-à-dire de contemplation de la nature et de rencontre avec une population locale sur un mode sensible, lent et intime. On peut donc considérer le voyage aventure comme un bien d'évasion dans le sens où le client est l'artisan du produit qu'il consomme. Son état d'esprit et son sentiment de vivre une expérience particulière lui permettent de maintenir un niveau de satisfaction élevé (Caccomo et Solonandrasana, 2006).

À partir des années 1990, l'attrait pour le voyage aventure a entraîné la constitution d'un espace de production autour de quelques agences emblématiques: Terres d'Aventure, Explorator, Club Aventure, Nomade Aventure, Atalante, Désert, Allibert. La recherche de distinction est son moteur essentiel (Passavant, 1999). À mesure qu'ils accumulent des expériences touristiques et sont plus nombreux, les clients deviennent plus exigeants. À côté des destinations devenues des classiques, comme les déserts d'Afrique du Nord ou les vallées du Népal, les agences sont contraintes d'innover, de rechercher l'inédit. Être un pionnier sur une destination ou se présenter comme le spécialiste d'une région du monde est un avantage concurrentiel primordial, déclare R. Dompier, cofondateur, codirecteur et responsable de communication chez Tirawa (2003 : communications personnelles). Du coup, 
Tableau 1: Les agences françaises de tourisme d'aventure

\begin{tabular}{l|l|l|l}
\hline Akaïna & Allibert & Atalante & La Balaguère \\
\hline Club Aventure & Explorator & Grand Angle & $\begin{array}{l}\text { Hommes } \\
\text { et Montagnes }\end{array}$ \\
\hline $\begin{array}{l}\text { Horizons } \\
\text { Nomades }\end{array}$ & Nomade Aventure & $\begin{array}{l}\text { Nouvelles } \\
\text { Frontières }\end{array}$ & $\begin{array}{l}\text { Odyssée } \\
\text { Montagne }\end{array}$ \\
\hline Tamera & Terres d'Aventure & Tirawa & UCPA \\
\hline Zig-Zag & \multicolumn{3}{l}{} \\
\hline \multicolumn{5}{|l}{ Source : compilation des auteurs. }
\end{tabular}

Source : compilation des auteurs.

Tableau 2: Les agences kirghizes de tourisme d'aventure

\begin{tabular}{l|l|l|l}
\hline Ak-Sai Travel & Alltournative & Alpatravel & The Alpine Fund \\
\hline Alp Tour Issyk Köl & AR Trek & Asiarando & Asia Mountains \\
\hline $\begin{array}{l}\text { Aventures et } \\
\text { Montagnes }\end{array}$ & $\begin{array}{l}\text { Celestial Moun- } \\
\text { tains }\end{array}$ & $\begin{array}{l}\text { Kyrgyz Community } \\
\text { Based Tourism } \\
\text { Association }\end{array}$ & Compass \\
\hline Dostuck Trekking & Fantastic Asia & ITMC Tien-Shan & Kirgiz Travel \\
\hline Kyrgyz Concept & $\begin{array}{l}\text { Maison du } \\
\text { Voyageur }\end{array}$ & Muza Travel & Néofit \\
\hline Nomad's Land & Novinomad & PSI Turkestan & $\begin{array}{l}\text { Ultimate } \\
\text { Adventure }\end{array}$ \\
\hline Seller Adventure & $\begin{array}{l}\text { Shepherd's Way } \\
\text { Trekking }\end{array}$ & Tien-Shan Travel & Top Asia \\
\hline
\end{tabular}

Source : compilation des auteurs.

ce marché de niche est très fragile. Il existe un renouvellement important de petites structures (moins de 10 salariés) qui peinent à atteindre un seuil de rentabilité. Pour durer, elles doivent trouver un équilibre délicat entre des destinations classiques, peu coûteuses mais à faible rendement symbolique, et des nouveautés risquées mais attractives. Les effets délétères de cette concurrence ont été exacerbés par l'accroissement des coûts de production et notamment ceux du transport aérien et par un contexte peu favorable au tourisme international (épidémies, guerres, irruption volcanique, etc.). Ils ont entraîné une transformation profonde des conditions de production du voyage aventure.

Les fondateurs des premières agences se présentaient comme des artisans qui vendaient leurs propres manières de voyager et choisissaient les nouvelles destinations sur la base de ce qu'ils qualifiaient de "coups de cour» personnels (Passavant, 2002). Ils maîtrisaient tous les éléments de la chaîne de valeur des voyages (Porter, 1982) et accompagnaient même parfois des groupes de clients. Aujourd'hui, la fabrication du voyage (durée, itinéraire, étapes), l’accueil et l'encadrement des clients, la fourniture de la logistique (matériel de camping, moyens de déplacement, vêtements adaptés, médicaments) sont délocalisés et confiés à des agences implantées localement, appelées réceptifs ou agences réceptives. Comme dans l'industrie, les différentes activités de la chaîne de valeur connaissent une nouvelle répartition spatiale (Porter, 1982). Les agences françaises définissent le produit et le mettent sur le marché. Pour le reste, elles ont recours à une kyrielle de sous-traitants : "Celui qui vend n'est plus celui qui fabrique», souligne Y. Briand, directeur de production chez Club Aventure (2003 : communications personnelles). Le voyagiste, c'est-à-dire «le fabriquant de voyage $[\ldots]$ maîtrisant tous les éléments du séjour, n'existe plus» (Mill, 2003 : communications personnelles). Cette stratégie d'internationalisation, entendue comme l'établissement de relations commerciales avec des partenaires étrangers (Johanson et Vahlne, 1990; Lehtinen et Penttinen, 1999), permet une réduction des coûts. Non seulement l'agence française a moins de capitaux financiers immobilisés pour le matériel et un encadrement moins coûteux (Chan et al., 1995), mais aussi elle diminue ses risques si la destination n'attire pas suffisamment de clients. Son activité se concentre alors sur l'identification des attentes émergentes de la clientèle pour définir un produit adapté (Caccomo et Solonandrasana, 2001) et sur le choix du prestataire.

Dans chaque pays, la multiplication des sous-traitants favorise l'émergence de relations de concurrence. En fonction de leurs atouts, ils vont alors occuper des positions différentes au sein d'un espace de production. Notre réflexion s'inscrit dans la problématique générale des relations entre différents espaces nationaux de production touristique et de leurs articulations. Il ne s'agit ni de dénoncer les effets néfastes de la diffusion des modèles culturels et économiques occidentaux, ni de valoriser les capacités de résistance identitaires de minorités culturelles soumises à l'hégémonie occidentale. L'interaction touristique ne peut se réduire «à la confrontation de deux entités culturelles dont chacune tenterait de tirer parti de l'autre» (Géraud, 2002 : 447). Nous adopterons une perspective constructiviste pour examiner les conditions de production de cultures touristiques en interrogeant la manière dont les offres se développent en fonction de particularités nationales. Ainsi, notre réflexion s'articulera autour de l'hypothèse centrale d'une homologie structurale entre l'espace des agences françaises et celui de leurs sous-traitants (Bourdieu, 1992). Il s'agit de vérifier si les agences les plus prestigieuses et les plus distinctives font bien appel aux correspondants les plus reconnus et les plus inventifs tandis que les agences plus confidentielles ont recours à des correspondants moins bien implantés localement.

Pour mieux saisir ces mécanismes, nous nous concentrerons sur une destination récente du tourisme d'aventure, le Kirghizstan. En effet, ce pays rassemble toutes les caractéristiques du produit valorisé dans la logique distinctive du tourisme d'aventure. Fermé au tourisme jusqu'à une date récente (1991), il est encore méconnu du grand public tout en présentant les ressources naturelles et culturelles les plus importantes de la région centre-asiatique, qui rassemble également le Kazakhstan, l'Ouzbékistan, le Tadjikistan et le Turkménistan. L'enquête de terrain a été conduite en deux temps. Tout d'abord, nous avons rassemblé des données 
ILLUSTRATION 1 : Yourtes kirghizes au bord du lac Song-Köl (photo : Julie Hallé).

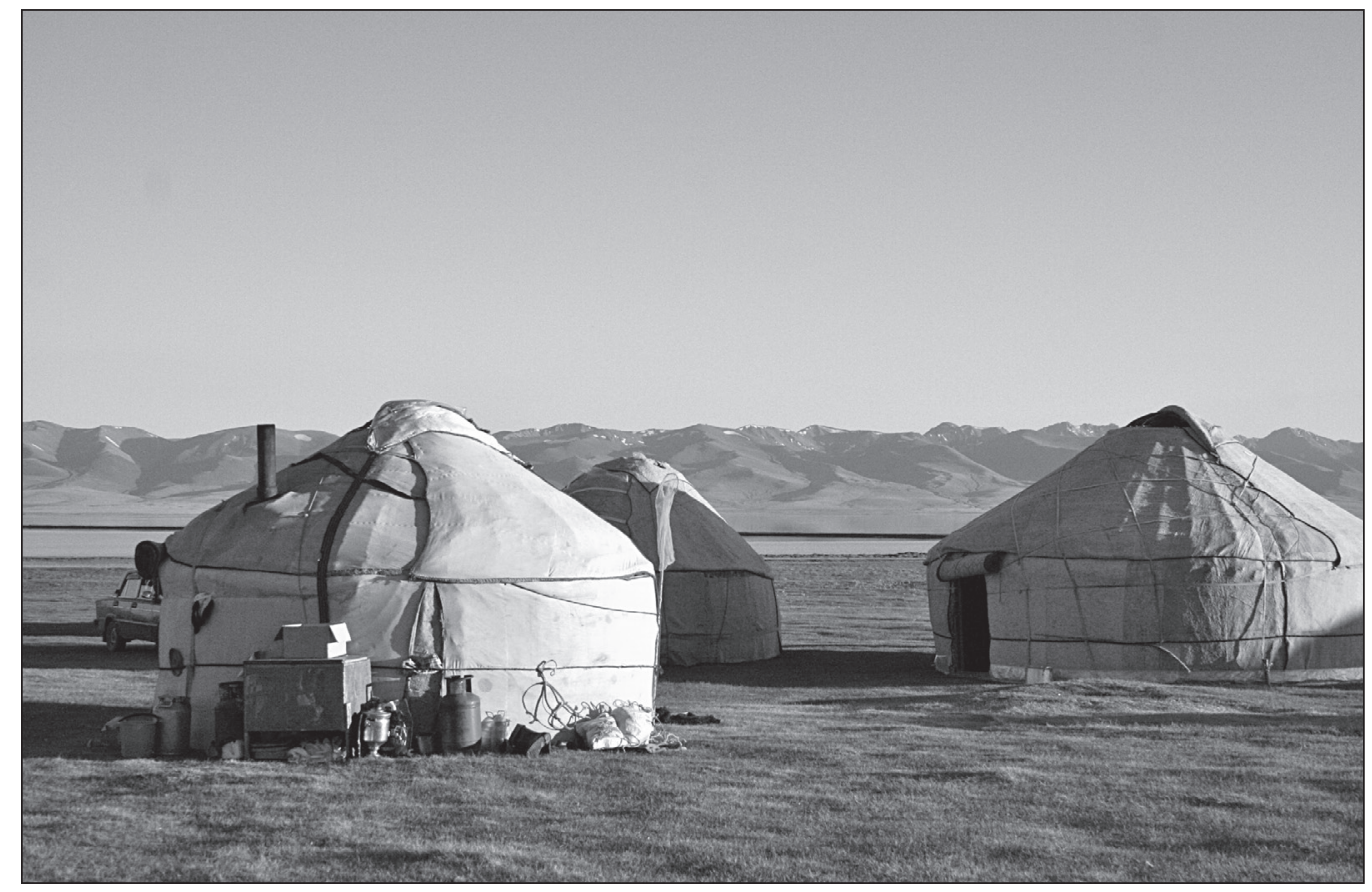

auprès des voyagistes français à partir de leurs brochures, de leurs sites sur Internet et d'entretiens avec leurs responsables ou des responsables de production. L'échantillon comporte les agences dont les productions sont les plus importantes (voir tableau 1).

Ensuite, nous avons identifié les caractéristiques des destinations kirghizes et les formes de consommation proposées. Les indicateurs utilisés sont principalement qualitatifs : lieux visités, vocabulaires utilisés, manière de présenter les populations locales, type de consommation suggérée. Nous avons également effectué 28 entretiens semi-directifs auprès de différents agents du tourisme d'aventure kirghizes au cours de plusieurs séjours réalisés entre 2003 et 2006 (voir tableau 2).

Les entretiens, d'une durée moyenne de deux heures, se sont déroulés majoritairement sur les lieux de travail des acteurs interrogés, permettant ainsi l'accès au fonctionnement quotidien de l'entreprise. Le corpus a fait l'objet d'une analyse de contenu (Bardin, 1993) permettant de révéler les modes d'organisation des agents interrogés, leurs stratégies dans l'espace de production local et de faire le lien avec leurs trajectoires professionnelles personnelles. Tout le travail de recueil des données empiriques, en France comme au Kirghizstan, a été réalisé par Julie Hallé dans le cadre de sa thèse de doctorat dirigée par le professeur Michel Raspaud (Hallé, 2008).

Après l'identification des grandes caractéristiques de l'offre des voyagistes français, nous verrons qu'elle a été façonnée par trois générations successives d'entreprises kirghizes. Les données rassemblées sur place révèlent que les réceptifs privilégiés par les agences françaises ont été fondés par des compatriotes expatriés. Nous verrons les avantages concurrentiels de cette option avant d'analyser les stratégies et les ressources mobilisées par les expatriés pour s'implanter durablement dans cet espace de production.

\section{L'offre des voyagistes d'aventure français au Kirghizstan}

Le Kirghizstan est régulièrement comparé à la Suisse de l'Asie centrale. Il jouit de nombreux attraits touristiques, c'est-àdire de ressources naturelles et culturelles qui peuvent rendre une destination attrayante pour un touriste (De Grandpré, 2007 : 16). C'est un petit pays à faible densité de population : $198000 \mathrm{~km}^{2}$ pour 4,5 millions d'habitants. Sa topographie est essentiellement montagneuse puisque $94 \%$ de son territoire est recouvert par des massifs montagneux et que son altitude moyenne est de 2750 mètres. Les sommets les plus hauts se situent dans la chaîne de montagnes des Tien Shan (Pic Pobedy, 7439 m; Pic Kan Tengri, 7010 m) et du Pamir Altaï (Pic Lénine, $7134 \mathrm{~m}$ ). Ces sommets encadrent de larges vallées, des hauts plateaux et le lac Issyk Kul, deuxième lac le plus haut du monde après le lac Titicaca, au Pérou.

Au niveau culturel, c'est un pays qui comporte une grande diversité ethnique, avec notamment des familles au mode de vie semi-nomade. Riche en sites historiques puisqu'il est traversé par l'ancienne Route de la Soie, il a conservé quelques édifices remarquables : anciennes cités caravanières (ex. : Caravansérail de Tash Rabat, XV siècle), minarets (ex. : Tour de Burana, $\mathrm{XI}^{\mathrm{e}}$ siècle), mosquées, etc.

Le dynamisme du tourisme kirghiz n'est pas récent. Durant l'époque soviétique, il était concentré prioritairement autour du lac Issyk Kul avec un tourisme balnéaire et de repos dans des pensionnats et des sanatoriums. Le tourisme de montagne est également bien ancré, avec des camps d'alpinisme aux pieds des principaux sommets. Le Pic Lénine, considéré comme le plus haut sommet d'URSS avant d'être précisément mesuré en 1933, est présenté comme un des 7000 mètres les plus accessibles et donc les plus gravis au monde (Jouty et Odier, 1999 : 412). Le Kirghizstan accueillait 


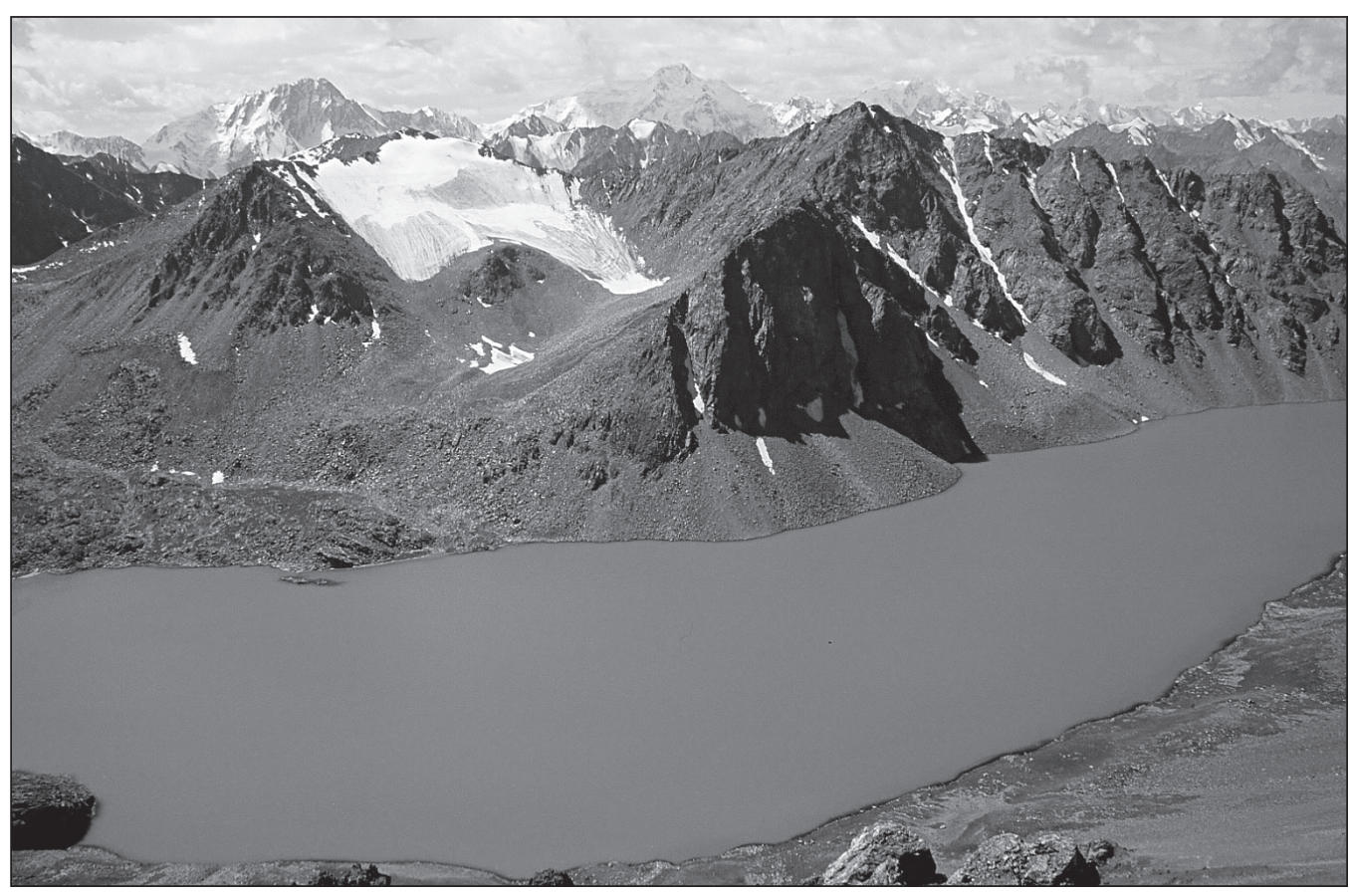

ILLUSTRATION 2 : Lac Ala-köl, massif des Tian-Shan central (photo : Julie Hallé). un nombre important de touristes, provenant presque exclusivement de l'URSS, avec une grande majorité de Russes et de Kazakhs. Le tourisme répondait aux contraintes idéologiques dictées par Moscou sur l'organisation des temps de travail et de loisir du citoyen soviétique. Il était encadré par différentes structures étatiques selon les types de publics. L'accueil des étrangers (hors URSS) était autorisé, mais rare et exclusivement organisé par l'intermédiaire de l'agence Intourist, créée en 1929, qui disposait de ses propres infrastructures (hôtels, transports, personnels, lieux de séjours, etc.). Les circuits touristiques des étrangers étaient ainsi séparés de ceux des résidents de l'Union. Les jeunes Soviétiques avaient leur propre agence de voyages, Spoutnik, sous l'égide directe du Komsomol (organisation de la jeunesse communiste liée au Parti communiste). Enfin, les syndicats organisaient les sorties touristiques et sportives des travailleurs.

La politique de perestroïka a permis une plus grande ouverture du pays au tourisme international, caractéristique d'une «transition territoriale» (Thorez, $2006: 65$ ). Cela s'est traduit par une simplification des formalités d'obtention des visas et des procédures d'enregistrement touristiques, mais aussi par un désenclavement géographique, notamment avec l'augmentation des liaisons aériennes avec le reste du monde. Dès 1988, le Kirghizstan apparaît dans le catalogue du voyagiste Terres d'Aventure, qui possède une position de leader dans l'espace de production français (Passavant, 1999). Initialement, la destination est valorisée pour son potentiel alpin avec des expéditions au sommet du Pic Lénine. Trekking et voyages de découverte ne seront proposés qu'après l'indépendance du 31 août 1991.

Dans les brochures françaises actuelles, le Kirghizstan est la destination d'Asie centrale la plus fréquente. Elle concentre plus de la moitié de l'ensemble des itinéraires proposés. Les circuits proposent des liaisons avec les pays voisins et prennent des formes multiples : trekking, véhicule $4 \times 4$, découverte, alpinisme, vélo de montagne (ou vélo tout-terrain), randonnée équestre, voyage en famille. La majorité des séjours ont une durée de 16 à 21 jours, et la gamme tarifaire moyenne se situe autour de 2500 euros pour deux semaines. Les différents supports de communication des voyagistes (brochures, descriptifs, fiches techniques, photos) déclinent l'offre kirghize autour de quatre attractions, c'est-à-dire des attraits mis en valeur à des fins touristiques (De Grandpré, 2007 : 16). La première, qui est aussi la plus répandue, c'est la culture nomade, souvent comparée à celle de la Mongolie (voir illustration 1).

L'archétype du nomadisme est représenté par des espaces naturels (paysage de steppes), un habitat traditionnel (yourtes), une utilisation du cheval comme moyen de déplacement, des activités pastorales et des pratiques culturelles (la chasse à l'aigle, la fabrication du feutre (shyrdak: tapis de feutre), les spécialités culinaires comme le kymys (koumis), qui est un lait de jument fermenté, les boorsok (petits morceaux de pâte frite) ou les kuruts (boulettes de yaourt séchées)). Les brochures proposent de vivre avec les nomades, de partager des moments de leur vie quotidienne. Elles promettent la découverte d'un mode de vie, de coutumes, un accès direct et sans artifice à une culture ancestrale présentée comme encore vivace.

La seconde attraction renvoie aux ressources naturelles du pays qui permettent la pratique de trekkings plus sportifs et de l'alpinisme (voir illustration 2). On trouve ainsi des titres évocateurs comme celui du catalogue d'Atalante en 2008, qui intitule son séjour L'Himalaya kirghiz.

La troisième attraction est construite à partir du rattachement du Kirghizstan à la célèbre Route de la soie, qui véhicule un imaginaire fort, amplement utilisé dans les circuits combinés (voir illustration 3 ). 
ILLUSTRATION 3 : Caravansérail de Tash Rabat (photo : Julie Hallé).

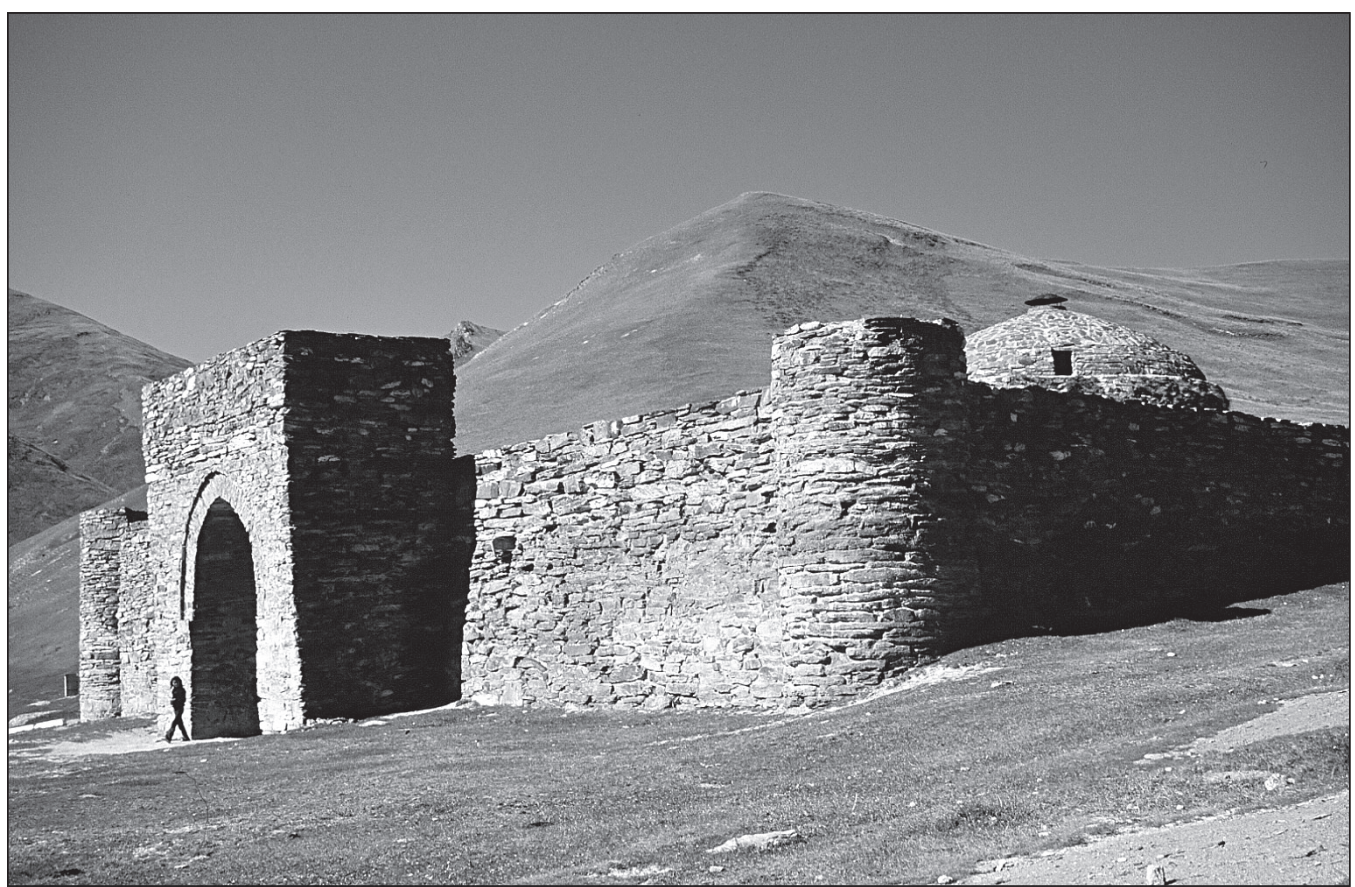

Les descriptions évoquent, non sans lyrisme, «... une artère vitale qui depuis des siècles lie l'Orient à l'Occident, la route de toutes les convoitises, de tous les rêves, de tous les conquérants...» (Explorator, 2010). Elles mobilisent tout un univers symbolique autour de personnages emblématiques, comme Gengis Khan, Manas, Alexandre le Grand (Terres d'Aventure, 2011) ou Marco Polo (Explorator, 2010). Les circuits traversent les sites remarquables qui manifestent un passé glorieux dont il s'agit de retrouver les traces.

Steppes, canyons, hautes vallées alpestres, lacs aux eaux turquoises... Contraste entre les simples toiles de feutre des yourtes installées pour l'été par les nomades kirghizes et l'architecture multiséculaire des villes mythiques de Khiva, Boukhara et Samarcande. Voyage à la rencontre de deux cultures : nomades de l'Asie Centrale, descendants de Gengis Khan, et bâtisseurs, fils de Tamerlan (Allibert, 2011).

Enfin, la quatrième attraction, plus marginale, renvoie au caractère exploratoire, insolite et préservé de cette destination. Le thème de l'isolement est le plus récurrent. Il est présenté comme la garantie d'un environnement qui n'est pas soumis aux agressions de la modernité (pollution, bruit, vitesse) et du tourisme de masse (Zig-Zag, 2011). Ces différentes attractions touristiques ont été élaborées progressivement avec le développement de l'espace de production du tourisme kirghiz.

\section{Les logiques d'évolution des trois générations d'entreprises kirghizes de tourisme d'aventure}

L'effondrement du bloc soviétique en 1991 fait entrer le pays dans une période de transition socio-économique et politique (Andreff, 2007). Il a contraint le gouvernement à mettre en place de nouvelles institutions et de nouvelles lois régissant une activité touristique récemment commerciale, ce qui a favorisé l'émergence de nouveaux acteurs. Le tourisme d'aventure kirghiz s'est constitué en espace de production avec trois générations d'entreprises qui se sont accumulées (Hallé, 2008). Chacune d'elles est constituée d'agents possédant des ressources différentes qui vont déterminer leurs stratégies d'implantation.

Les premières entreprises ont été créées dès la perestroïka par des alpinistes chevronnés formés à l'époque soviétique. Profitant d'un processus de "privatisation chaotique» au moment de la transition libérale (Durand, 2005), ce petit groupe va acquérir à des prix dérisoires des bases matérielles (moyens de transport adaptés à la montagne, matériel d'alpinisme) et des infrastructures importantes (refuges, camp de base en montagne, base touristique). Ils vont s'enrichir rapidement grâce à ce capital technique difficilement remplaçable et constituer une nouvelle élite d'entrepreneurs. Ce sont essentiellement des membres de la nomenklatura ou de l'intelligentsia technique et scientifique. Ils sont très diplômés (Andreff, 2007). Ces propriétés sociales et culturelles leur ont permis de saisir immédiatement les opportunités offertes par l'indépendance. Plutôt tournés vers un tourisme sportif et alpin, ils se sont spécialisés dans l'accueil des groupes étrangers et ont offert aux premières agences françaises de nouvelles opportunités à travers cette destination inédite.

La fin des années 1990 constitue une époque charnière marquée par une plus grande ouverture du pays au tourisme international et la création d'une dizaine d'agences spécialisées. L'offre se diversifie avec un tourisme plus culturel et moins centré sur l'ascension de sommets. Des circuits de randonnée et des trekkings à pied, à cheval, en canoë ou en vélo de montagne apparaissent. Cette orientation bénéficie de l'impulsion d'organisations internationales qui importent une idéologie de préservation culturelle de la société kirghize 
(Pabion-Mouriès, 2011). Elle s'enracine rapidement dans ce contexte post-soviétique et multiethnique où la construction d'un État-nation se fait par l'émergence d'une identité nationale et l'exaltation d'un passé national. Ces nouvelles entreprises maintiennent une logique de spécialisation : le cheval pour les unes, le trekking ou l'eau vive pour les autres. Leurs responsables sont pour l'essentiel des guides accompagnateurs ayant exercés dans les entreprises de la première génération. La connaissance des sentiers de montagne, l'expérience acquise et intériorisée du marché leurs permettent de dépasser les produits existants et d'innover. À l'instar de leurs prédécesseurs, ils sont pour la majorité diplômés de l'université, sans pour autant être spécialisés dans le tourisme. C'est également le début des premières agences créées par des étrangers.

Enfin, les années 2000 ont vu une augmentation considérable du nombre et de la forme des agences produisant du tourisme d'aventure. Nous avons recensé plus d'une douzaine d'entreprises et d'associations, locales et internationales, ce qui transforme progressivement la structure et le fonctionnement de l'espace de production. Cette troisième génération étend son offre vers un tourisme plus individualisé et plus localisé. Elle est influencée par la présence d'organisations internationales non gouvernementales (ONG) qui ont pour objectif le développement global de la société kirghize et utilisent le tourisme comme moyen d'action. C'est le cas pour le projet de Community Based Tourism (CBT), soutenu par l'organisation suisse Helvetas, destiné à développer les pratiques touristiques des populations locales (Pabion-Mouriès, 2012). Les circuits sont moins sportifs, plus courts; les déplacements sont plus souvent véhiculés. Les agences s'adressent désormais à une clientèle de particuliers, recrutés par Internet. Ces entrepreneurs ont les mêmes propriétés sociales que ceux de la génération précédente, dont certains sont issus. On assiste en revanche à une plus forte présence d'expatriés et à un rajeunissement de l'âge moyen (entre 25 et 35 ans). Parallèlement, le tourisme de montagne est relancé avec notamment la réhabilitation des camps d'alpinisme de l'ex-URSS par les Fédérations d'escalade et d'alpinisme, l'organisation du sommet international de la montagne à Bichkek en 2002 et le Raid Gauloise en 2003. Toutefois, l'instabilité politique depuis la révolution des Tulipes, en 2005, entraîne de multiples remaniements ministériels qui ont des conséquences négatives sur l'essor du tourisme.

L'espace de concurrence kirghiz du tourisme d'aventure s'est développé autour de quatre logiques :

1) L'innovation comme moteur principal. Tout comme en France, proposer une nouvelle destination ou une nouvelle forme de déplacement est un moyen de créer un avantage concurrentiel, de renouveler l'intérêt de la clientèle existante ou de conquérir une nouvelle clientèle. Elle permet de négocier des exclusivités avec les correspondants français.

2) Le glissement d'une production à dominante sportive vers des aspects plus culturels. Les premières agences ne proposaient que des randonnées, du trekking et de l'alpinisme, déjà bien implantés à l'époque soviétique. Les suivantes se sont plus tournées vers la découverte des traditions et des modes de vie, la rencontre avec les populations locales, des visites de sites historiques, en mobilisant différents moyens de déplacement. Cette évolution a deux avantages. En diminuant l'intensité de la dépense physique nécessaire, elle ouvre le voyage à une clientèle plus large. Elle permet également de différencier la destination en faisant référence à un univers symbolique fort et aisément identifiable par la clientèle occidentale : la route de la soie, le nomadisme, les peuples mongols, la nuit en yourte, etc.

3) Le passage d'un tourisme de groupe vers un tourisme plus individuel et sur mesure. Les premiers réceptifs se sont rapidement imposés en proposant des forfaits totaux à des groupes constitués par les agences étrangères. À partir des années 2000, les nouveaux réceptifs ont personnalisé les voyages en s'adressant à une clientèle dite d'individuels. Ce sont des petits groupes, à partir de deux personnes, adressés par les correspondants mais surtout recrutés par le biais d'Internet. Ils peuvent modifier tous les aspects du voyage : durée, activités, lieux visités, type d'hébergement, temps de déplacements, etc. Ce fonctionnement à la carte nécessite une grande souplesse de gestion que ne peuvent s'autoriser que les petites structures connaissant bien le pays.

4) Le maintien d'une logique de spécialisation généralisée. La spécialisation sur un type de produit est un moyen pour les agences kirghizes de créer une valeur ajoutée (Pons et de la Fouchardière, 2003) et de limiter ou, tout du moins, de neutraliser la concurrence. Cela provoque un cloisonnement de l'espace de production laissant apparaître trois formes de pouvoir : celui lié à la possession d'une base matérielle et des compétences techniques nécessaires pour l'encadrement de l'alpinisme, celui lié à la connaissance d'une région peu couverte par les autres agences et celui lié à la maîtrise d'un moyen de déplacement original, comme l'héliski, les sports d'eau vive ou la randonnée équestre. Cela entraîne également une interdépendance entre les agences réceptives. Ainsi, les premières agences, contraintes de suivre les évolutions du tourisme d'aventure kirghiz, ont sous-traité une partie de leurs engagements à d'autres réceptifs qui disposaient de la base matérielle nécessaire ou à des guides connaissant la région en question. Les agences sont alors liées par des échanges commerciaux croisés multiples et les maillons de la chaîne de valeur s'allongent, ce qui rend plus délicat, pour l'agence étrangère, le contrôle de la qualité de la prestation.

Ainsi, l'espace de production du tourisme d'aventure kirghiz est dominé par les premières entreprises qui ont accumulé les ressources les plus importantes : un capital symbolique de notoriété lié à leur ancienneté, un important capital social qui se caractérise par des relations étroites avec les membres du gouvernement et un capital technologique constitué par des infrastructures importantes, acquises lors de la perestroïka. Contraint à l'innovation pour exister, les nouveaux entrants ont diversifié l'offre touristique. Néanmoins, certaines entreprises centre-asiatiques conservent des caractéristiques de l'époque soviétique : sousemploi, absence de responsabilisation des travailleurs, faible crédibilité des instances dirigeantes, etc. Pour beaucoup, la notion de service vis-à-vis des fournisseurs et surtout celle de client sont encore vagues. 


\section{Le choix des sous-traitants : la confiance va aux expatriés}

Pour Torrès (dans Botti et al., 2008 : 32), la gestion des PME se caractérise par la proximité : proximité hiérarchique dans la centralisation de la gestion, proximité intrafonctionnelle dans la faible spécialisation des tâches, proximité temporelle dans les stratégies implicites et à court terme et proximité spatiale dans le choix de marchés proches. Comment les agences françaises sélectionnent-elles leurs correspondants étrangers? Comment introduisent-elles la proximité nécessaire à l'établissement d'une relation de confiance malgré la distance géographique? L'enquête de terrain révèle que les voyagistes français ont majoritairement la même stratégie : ils utilisent le plus souvent possible les services d'agences réceptives créées au début des années 2000 par des expatriés français. Nous allons revenir sur les caractéristiques de ces entreprises (voir tableau 3), sur les caractéristiques de cette proximité et sur les conditions d'implantation des expatriés français dans un contexte peu favorable.

La première agence à travailler avec la France, l'agence Bleu, est devenue dominante en vendant des circuits clés en mains, entièrement conçus par son fondateur :

Je leur vends tout... je leur donne même les photos. Je leur fais la fiche technique... je fais tout de A à Z. Et puis, je vais par exemple chez Allibert faire une présentation du pays aux vendeurs, à Club Aventure, à Zig-Zag, à Nomade. Je leur montre des CD, pour leur présenter la destination, pour que les vendeurs puissent vendre la destination (Anonyme, 2006a : communications personnelles).

Le fondateur a réussi à capter la clientèle française en accueillant des groupes constitués, qui représentent environ $70 \%$ de son activité selon lui. Il faut dire qu'au moment de la création de son entreprise, les difficultés d'accès au Kirghizstan favorisaient les voyages en groupe puisqu'il était plus facile de sécuriser et d'organiser une prise en charge collective pour l'obtention de permis de trekking, d'autorisation de circulation, de visas, etc. L'entreprise a investi une large zone géographique puisqu'elle propose des prestations dans toute la région centre-asiatique par des partenaires locaux. Initialement portée sur l'eau vive (principalement le rafting), les séjours se sont rapidement structurés autour du trekking et des voyages de découverte. Néanmoins, un renouvellement des produits est proposé chaque année par l'intégration de régions encore inexplorées ou de nouvelles activités sportives ou culturelles comme l'héliski, le vélo de montagne, la randonnée équestre, la visite d'un site historique. Les autres agences se sont positionnées ensuite en adoptant une stratégie de différenciation marquée par rapport à Bleu, tant dans le type de circuit que dans la forme de consommation proposée.

La seconde entreprise, Vert, a été créée en 2001 par un couple de Français avec leurs deux enfants, de la jeune vingtaine. Le fondateur est décédé en 2007, mais sa femme et ses enfants poursuivent l'activité. Ils vivent toute l'année au Kirghizstan. L'idée de s'installer dans le pays et de fonder cette agence est venue du père qui, en 1999, a eu un contrat comme volontaire pour l'ONU au Kirghizstan pendant deux ans. Depuis 2005, ils ont développé une entreprise similaire en Uruguay. Ils y sont propriétaires d'un voilier. Cela leur permettrait un éventuel repli stratégique en fonction de l'évolution politique et sociale du Kirghizstan. Ces entrepreneurs passionnés positionnent leur entreprise sur le tourisme équestre. Ils sont les principaux partenaires du leader français des voyages équestres : Rando Cheval. Premiers et longtemps seuls sur ce créneau, ils visent des prestations de qualité en garantissant les départs à partir de deux personnes et un logement exclusif chez l'habitant. Ils encadrent eux-mêmes les circuits, proposent une infrastructure et une base matérielle solides et adaptés aux standards français (45 chevaux équipés, entretenus et soignés selon les normes occidentales). Néanmoins, avec l'augmentation de la concurrence locale sur le marché du tourisme

Tableau 3: Les sous-traitants expatriés des voyagistes français

\begin{tabular}{|c|c|c|c|c|c|}
\hline $\begin{array}{l}\text { Agence (noms modifiés } \\
\text { pour maintenir l'anonymat) }\end{array}$ & Année de création & Clientèle & Origine de la clientèle & Partenariats français & Types de prestation \\
\hline Bleu & 1999 & $\begin{array}{l}70 \% \text { groupes } \\
30 \% \text { individuels }\end{array}$ & $\begin{array}{l}90 \% \text { France } \\
10 \% \text { Suisse et Canada }\end{array}$ & \begin{tabular}{|l|} 
Allibert \\
Arvel \\
Club Aventure \\
Nomade Aventure \\
Nouvelles Frontières \\
UCPA \\
Zig-Zag
\end{tabular} & $\begin{array}{l}\text { Multiples activités : } \\
\text { eau vive, trekking, vélo } \\
\text { de montagne, voyage } \\
\text { découverte... } \\
\text { Couverture géographique } \\
\text { large }\end{array}$ \\
\hline Vert & 2001 & $100 \%$ groupes & France et Suisse & Rando Cheval & $\begin{array}{l}\text { Randonnée équestre } \\
\text { Trekking }\end{array}$ \\
\hline Rouge & 2001 & $\begin{array}{l}50 \% \text { groupes } \\
50 \% \text { individuels }\end{array}$ & France & \begin{tabular}{|l} 
Explorator \\
Grand Angle \\
Terres d'Aventure \\
Tirawa
\end{tabular} & $\begin{array}{l}\text { Logement chez l'habitant, } \\
\text { vente de souvenirs, trek- } \\
\text { king, randonnée équestre, } \\
\text { voyage découverte }\end{array}$ \\
\hline Jaune & 2003 & $\begin{array}{l}50 \% \text { groupes } \\
50 \% \text { individuels }\end{array}$ & France & $\begin{array}{l}\text { Partenariats en cours de } \\
\text { négociation }\end{array}$ & $\begin{array}{l}\text { Trekking, randonnée à la } \\
\text { carte et haut de gamme }\end{array}$ \\
\hline
\end{tabular}

Source : compilation des auteurs. 
équestre et des destinations proches comme la Mongolie, l'entreprise tente de s'ouvrir à d'autres activités comme le trekking, mais elle souffre d'une image trop marquée.

L'agence Rouge a été créée en 2001 par une Kirghize mariée avec un Français. Structure familiale composée de quatre personnes, l'entreprise propose du logement chez l'habitant, du trekking, des voyages de découverte, un dépôt-vente de souvenirs kirghizes et plus récemment de la randonnée équestre dans plusieurs républiques centre-asiatiques. La fondatrice parvient à concevoir des voyages originaux dans des régions inédites et qui privilégient la proximité avec les habitants. Toutefois, leur base matérielle est peu développée et essentiellement constituée de produits achetés en France, chez Décathlon. Sa clientèle est exclusivement francophone, majoritairement française et jeune (de 20 à 45 ans).

Enfin, l'agence Jaune a été fondée en 2003 par un jeune couple franco-kirghiz d'une trentaine d'années. Au départ, ils souhaitaient offrir un soutien à leur réseau amical et familial pour l'organisation de trekkings. L'ouverture d'une structure commerciale s'est imposée dans un second temps. La stratégie de production se concentre sur la qualité, malgré une base matérielle peu développée, et sur une politique de prix attractifs. Les trekkings proposés sont confortables (pas de portage, logement de qualité, nourriture raffinée) et d'un niveau physique modéré, uniquement au Kirghizstan. L'agence est spécialisée dans une région peu fréquentée, les Terskey Alatau, au sud du lac Issyk Kul, région d'origine de la cofondatrice. La clientèle est composée essentiellement d'individuels francophones qu'ils recrutent par leur site Internet et par le bouche à oreille. Des partenariats sont en cours de négociation avec des agences françaises, mais nous n'avons pas pu savoir lesquelles. L'agence cherche à être au plus près des attentes des clients en proposant des séjours à la carte encadrés par le couple et son réseau familial.

Pour tous les sous-traitants, la qualité de la logistique (matériel, logement, transport, nourriture, encadrement par des guides compétents parlant l'anglais ou le français) est un élément important dans la relation de confiance qu'ils établissent avec l'agence française. L'entreprise Bleu l'a bien compris en offrant toutes les garanties d'un ajustement précis aux attentes de la clientèle française. Tout d'abord, le fondateur a réalisé d'importants investissements en construisant une guesthouse pour assurer le confort des groupes. Ensuite, il dispose d'une base matérielle conséquente, achetée en France, avec des équipements de camping (matelas, duvet, tentes techniques) et des vêtements (gants, vestes, pantalons) de marques réputées. Ils possèdent des véhicules adaptés aux terrains difficiles et des matériels techniques pour les activités sportives (chevaux, selles, vélos, crampons, cordes, etc.). Il a formé des guides locaux parlant le français et accompagne systématiquement les groupes sur un nouveau circuit afin de réaliser les ajustements nécessaires. Enfin, il se fournit en produits pharmaceutiques directement en France. Cette stratégie est délibérée : «Avant d'être au Kirghizstan, j'ai travaillé avec beaucoup d'agences au Népal, et un peu partout dans le monde. Je sais précisément ce que les gens attendent et ce qu'il faut mettre en place» (Anonyme, 2006a : communications personnelles). De cette façon, il renforce sa position de leader en offrant des garanties visibles du niveau des prestations proposées, directement mesurables par ses partenaires français qui connaissent les produits utilisés par les clients.

Traiter avec des expatriés français permet aux agences françaises de retrouver une proximité et de diminuer l'asymétrie de l'information. Ces sous-traitants connaissent à la fois les exigences de la clientèle française et les règles complexes d'un pays en situation de transition. De plus, l'un d'entre eux vit une partie de l'année en France, ce qui facilite les échanges commerciaux. Ils partagent la langue, la culture, la même «doxa» professionnelle (Bourdieu, 1979:60) : ils ont une complicité et une connivence fondées sur l'adhésion immédiate aux mêmes évidences, aux mêmes opinions, aux mêmes croyances. L'importance du capital social au sein de l'entreprise ainsi que les investissements réalisés (Glaeser, 2001) influent directement les mécanismes de confiance (Fafchamps, 2002) auprès des partenaires et sur la circulation de l'information (Truchet et al., 2008).

Toutes les agences françaises n'ont pas recours à des soustraitants expatriés. Alors, pour minimiser les risques vis-à-vis de la qualité du voyage et de la satisfaction des clients, elles doivent investir dans la connaissance du pays et dans un contrôle plus fort du réceptif local, par exemple en envoyant un accompagnateur français, ce qui est plus coûteux.

\section{Les conditions d'accès des expatriés à l'espace de production kirghiz du tourisme d'aventure}

Le contexte de transition de la république kirghize a créé des conditions particulières d'implantation pour les entreprises touristiques. La réglementation n'impose pas de règle spécifique pour un étranger qui souhaite ouvrir une firme touristique. Il n'a donc aucune obligation de s'associer à un tiers kirghiz hormis pour l'acquisition de terres. Sur ce point, il existe de nombreux obstacles bureaucratiques destinés à rendre l'acquisition des terres impossible aux étrangers. À travers ces réformes, le gouvernement kirghiz a transformé le régime foncier d'un monopole étatique hérité du système soviétique en un système quasi privé. Depuis l'indépendance, il a certes individualisé le régime foncier du pays (Lerman, 2009) puisqu'il a mis en place des droits privés d'utilisation et de disposition ainsi que la possibilité d'hypothéquer ces droits, mais celui-ci comprend toujours une possession étatique des terres (Terauds, 2010). Cela rend concrètement impossible l'acquisition par un étranger sans l'intermédiaire d'un tiers kirghiz. Ce système de lois foncières et de droits liés à la propriété donne parfois lieu à des stratégies de contournement et des pratiques informelles (Désert, 2006) chez les entrepreneurs touristiques. Dans le contexte local, «le principe de la propriété, fondamental dans la logique capitaliste, est neutralisé par le système des protecteurs (Krycha - Крыша). Les différents systèmes de protection informelle (aussi bien politique que mafieuse) auxquels ont recours les entrepreneurs pour se prémunir tout autant de l'incertitude juridique que de l'insécurité encadrent finalement l'usage de la propriété» (Désert, 2006 : 18). Si elles veulent réussir leur intégration, les entreprises touristiques gérées par des étrangers sont contraintes de s'entourer de personnes ressources afin de pouvoir accéder à la propriété et développer leurs activités commerciales. 
Même si l'entreprenariat dans le domaine touristique ne semble pas contraint, hormis pour l'acquisition foncière, les entretiens ont révélé chez l'ensemble des acteurs interrogés l'adoption d'une stratégie de discrétion. Les entreprises ont leurs sièges sociaux dans des appartements ou des maisons privées. L'entreprise Bleu, par exemple, n'a pas de devanture particulière et n'a recours à aucune démarche publicitaire. Elle n'est pas mentionnée dans les guides de voyages; elle n'a aucun catalogue et n'est pas dans l'annuaire. Elle ne fait pas non plus de communications localement, excepté par son site Internet. Globalement, les agences n'ont pas besoin de comptoir commercial pour l'accueil de leurs clients. Cette discrétion leur permet aussi d'échapper aux tracasseries d'une administration complexe.

Ici, au niveau de l'administration, c'est resté comme à l'époque soviétique. Si on a besoin de mettre un panneau, par exemple, il faut un permis d'architecture... il faut deux ou trois documents. Il faut faire verbaliser ton enseigne par un architecte d'État. Et en plus, ça te coûte assez cher. Pour un petit panneau, c'est 1400 soms par an et tu perds au moins quatre jours pour les formalités. Donc, les gens ne s'embêtent pas. Du coup, tout le monde reste dans les maisons [...] Ici, tout est difficile à trouver. Pour les guesthouses, il y en a beaucoup qui ne sont pas indiqués du tout. Comme ça, ils sont moins emmerdés par les impôts [...] De toute façon, ce sont les agences qui vont à la rencontre de leurs clients à l'aéroport. Donc, le touriste ne va jamais dans l'agence. Tu l'amènes à l'hôtel ou dans la montagne (Anonyme, 2006b : communications personnelles).

Après l'indépendance, dans l'optique d'une construction ou reconstruction des institutions politiques et économiques, le gouvernement kirghiz a fait le choix de l'ouverture internationale (Pétric, 2006). Dès lors, un grand nombre d'organisations internationales se sont installées sur le territoire dans le but de faire émerger une société civile (Pétric, 2007). Pour s'intégrer, les expatriés français investissent dans des projets de développement et dans des activités connexes. L'agence Bleu a créé une école de formation de guides locaux qui reprend les critères européens de formation en s'inspirant du Brevet d'État d'Accompagnateur en Moyenne Montagne. Elle collabore avec l'association Kochcorka, qui est une coopérative de femmes qui fabriquent des souvenirs kirghizes. Enfin, elle soutient la fondation Babushka Adoption, créée en 1999 avec le soutien financier de l'Agence suisse pour le Développement et la Coopération (DDC), qui a pour objectif d'améliorer les conditions de vie des personnes âgées vivant en situation d'isolement et de précarité au Kirghizistan. L'aide prend la forme d'un soutien financier, social et moral. Les donateurs peuvent adopter symboliquement une babushka ou un dedushka (mamie et papi en russe). L'agence Rouge complète son activité par un magasin de dépôt-vente de souvenirs kirghizes. Elle est en relation avec 44 artisans dans le pays, ce qui leur permet également de développer leur réseau pour le logement chez l'habitant. Jaune a fait le choix d'impliquer une grande partie de la famille de la cofondatrice, en leur fournissant des emplois saisonniers, tandis que Vert s'engage localement dans l'investissement et l'entretien des nombreuses montures. L'entreprise fournit des emplois à des paysans kirghizes. Ces engagements locaux sont encouragés par les agences françaises qui en font la promotion dans leurs catalogues en les présentant comme des actions de développement durable.

Enfin, pour réussir leur implantation, les expatriés français ont mobilisé un ensemble de ressources culturelles et sociales, issues de leurs trajectoires scolaires et professionnelles. Tous ces entrepreneurs sont jeunes, entre 35 et 45 ans, et hautement qualifiés. Ils possèdent des diplômes de l'enseignement supérieur de niveau master au minimum, français ou kirghizes (équivaut à quatre années d'études supérieures après le baccalauréat), majoritairement orienté sur l'économie internationale ou les langues étrangères (voir tableau 4).

Avant de s'installer, ils disposaient donc d'un capital international (Wagner, 1998 et 2007) accumulé lors de formations puis conforté par des expériences professionnelles à l'étranger. Ce capital international, à la fois scolaire et professionnel, est constitué de compétences entrepreneuriales (Chandler et Jansen, 1992), mais aussi de connaissances de l'environnement international (Reuber et Fischer, 1997). Il a favorisé la création d'entreprises qui répondent précisément aux attentes des agences françaises.

\begin{tabular}{|c|c|c|}
\hline Agence & Formation — Diplômes & Trajectoire professionnelle \\
\hline Bleu & $\begin{array}{l}\text { Maîtrise en sciences } \\
\text { économiques } \\
\text { MBA en gestion internationale } \\
\text { passé au Canada } \\
\text { Brevet d'État d'éducateur } \\
\text { sportif en ski alpin }\end{array}$ & $\begin{array}{l}\text { Pendant } 10 \text { ans, concepteur } \\
\text { de séjours au Canada, au } \\
\text { Chili, au Népal, en Argentine } \\
\text { pour l'UCPA } \\
\text { Pendant deux ans, } \\
\text { coresponsable d'une agence } \\
\text { qui propose des circuits de } \\
\text { descente de rivière } \\
\text { Moniteur de ski en France, } \\
\text { en hiver }\end{array}$ \\
\hline Vert & Diplôme d'ingénieur & $\begin{array}{l}\text { Expert international et } \\
\text { consultant pour l'ONU pendant } \\
\text { plusieurs années }\end{array}$ \\
\hline Rouge & $\begin{array}{l}\text { Elle : diplôme en Langues } \\
\text { étrangères de l'Université } \\
\text { de Bi } \\
\text { Lui : Bac technique }\end{array}$ & $\begin{array}{l}\text { Elle : pendant sept ans, } \\
\text { accompagnatrice puis } \\
\text { interprète et guide pour la } \\
\text { plus grosse agence kirghize } \\
\text { de randonnée } \\
\text { Lui : militaire, plusieurs } \\
\text { missions au Kirghizstan et } \\
\text { dans différents pays }\end{array}$ \\
\hline Jaune & $\begin{array}{l}\text { Elle : Maitrise d'économie } \\
\text { et diplôme de troisième } \\
\text { cycle universitaire (DESS) en } \\
\text { économie et développement à } \\
\text { I'Université de Grenoble } \\
\text { Lui : Ingénieur en informatique }\end{array}$ & $\begin{array}{l}\text { Elle : correspondante pour une } \\
\text { agence française } \\
\text { Lui : travailleur dans une } \\
\text { multinationale qui fabrique } \\
\text { du matériel audio-visuel et } \\
\text { informatique }\end{array}$ \\
\hline
\end{tabular}

TÉOROS, vol. 31, n 1, p. 103-114 @ 2012 
Par ailleurs, l'accumulation du capital social nécessaire à l'implantation dans l'espace de production kirghiz du tourisme d'aventure s'est fait de deux façons complémentaires : par le recours au réseau développé dans les activités professionnelles antérieures dans l'industrie touristique ou par des stratégies matrimoniales, ou par les deux. À l'instar de la société ouzbèke, le Kirghizstan accorde une grande valeur à la constitution et à la transmission du capital social (Pétric, 2002 : 5). Largement diffusé à l'époque soviétique, le seit (mot russe qui signifie «réseau» et qui vient de l'anglais set) reste essentiel aujourd'hui dans les relations économiques, par exemple pour obtenir une faveur ou une autorisation (Pétric, 2002 : 6). Considéré péjorativement comme du clientélisme dans les pays occidentaux, il constitue un "type de relation sociale caractérisé par des liens personnels et affectifs entre deux protagonistes qui disposent de ressources inégales et retirent un bénéfice mutuel de leur relation» (Pétric, 2002 : 195). Le réseau comporte donc une dimension économique mais aussi une dimension sociale : il donne lieu au Blat, qui est un système d'échange de biens et de services interentreprises informels, en vigueur dans tout le commerce de la zone centre-asiatique. L'accumulation de ce capital social particulier, à l'interface entre des relations d'ordre privé et des échanges économiques, est indispensable pour les entrepreneurs qui souhaitent s'implanter en Asie centrale. C'est une règle essentielle du fonctionnement de l'économie locale.

Le responsable de l'agence Bleu est celui qui a su le mieux structurer son réseau, en cumulant une connaissance des agences françaises, des contacts internationaux et des relations locales. Au départ, il y a son expérience professionnelle dans l'organisation de séjours en eau vive :

Cela ne se fait pas tout seul... Les premiers partenaires que j'ai eus, c'était l'UCPA parce que j'ai travaillé 10 ans avec eux au Canada sur des expéditions en rafting. Ils me connaissaient, ils connaissaient ma manière de travailler et mon expérience. Donc, ils m'ont fait confiance et on a travaillé ensemble sur la Kirghizie. À partir de là, tout s'est enclenché... À partir du moment où l'on travaille avec des agences comme l'UCPA, c'est une référence pour les autres dans le milieu. Je connaissais aussi des gens chez Allibert, et donc ils m'ont fait confiance et puis voilà. Après, les autres agences viennent te voir et te disent : "Nous, on est intéressés parce que la Kirghizie, c'est une nouvelle destination, on n'a rien pour l'instant, qu'est-ce que tu peux nous proposer?" Je leur ai monté des séjours et puis voilà, c'est parti comme ça (Anonyme, 2005 : communications personnelles).

Devenir partenaire de l'agence Allibert après avoir longtemps travaillé pour l'UCPA est tout à fait logique. Ces deux établissements ont le même positionnement dans l'espace du tourisme d'aventure en France : ils proposent des séjours avec une dominante sportive très affirmée. L'Union nationale des Centres sportifs de Plein Air a été fondée en 1965 sous l'impulsion de Maurice Herzog pour encourager la pratique d'activités physiques de pleine nature des jeunes adultes. L'agence Allibert a été fondée en 1987 par trois guides de haute montagne indépendants qui souhaitaient emmener des clients ailleurs que dans les Alpes. Ce positionnement plutôt sportif et l'exclusivité demandée par Allibert empêchent le fondateur de Bleu d'être partenaire de l'agence Terres d'Aventure, pourtant dominante dans l'espace de production français :

Moi, par exemple, je ne travaille pas avec Terres d'Aventure parce que j'ai travaillé avec Allibert au départ et ils m'ont demandé l'exclusivité. Les agences françaises, elles veulent bien travailler avec toi, mais si tu ne travailles pas avec les autres. Donc, moi, j'ai dis OK, même si, chez Terres d'Aventure, je connaissais pas mal de monde (Anonyme, 2006a : communications personnelles).

Enfin, il a été l'interlocuteur privilégié entre les organisateurs du Raid Gauloise, un événement sportif de portée internationale, le gouvernement kirghiz et les acteurs locaux. Il possède ainsi un positionnement symbolique fort marqué par une reconnaissance à la fois au niveau du pouvoir étatique, des partenaires locaux (agences touristiques) et des organisations internationales.

Le capital social peut également être accumulé par le biais d'une stratégie matrimoniale, dans le sens d'une alliance qui produit des effets objectifs qui sont différents des finalités subjectivement poursuivies (Bourdieu, 1972 : 1108). Les responsables des agences Rouge et Jaune ont épousé une Kirghize. Ils ont bénéficié d'un accès direct aux systèmes d'échanges et de profits matériels et symboliques de leur belle-famille. Ainsi, ils ne déclarent pas toujours les salaires versés à des guides ou des cuisiniers de confiance, membres de la famille élargie. Ils ont constitué un réseau de familles semi-nomades qui hébergent les touristes en été. Les circuits comportent par exemple des nuits dans les yourtes où les touristes partagent des activités pastorales avec les bergers, assistent à la fabrication du feutre, du kymys (koumis, lait de jument fermenté) et autres spécialités locales (kurut (boulettes de yaourt séchées), etc.). Ce capital social à base familial permet donc de proposer des produits distinctifs qui procurent aux clients le sentiment de rencontres plus authentiques avec les populations locales.

\section{Conclusion}

Le tourisme international se développe en suivant des processus complexes d'ajustement et de transformation. Il n'impose pas brutalement des pratiques et des idéologies homogènes à des groupes sociaux jusqu'alors éloignés de ces préoccupations. Comprendre ces processus nécessite d'adopter une perspective structurale, c'est-à-dire d'étudier l'émergence et l'évolution d'une offre touristique selon les contextes sociaux, économiques et politiques et d'analyser les caractéristiques de ses principaux acteurs dont la position dans cet espace de concurrence détermine les possibilités d'actions et les stratégies.

Le cas particulier du Kirghizstan montre comment une société en transition, marquée par une tradition de tourisme de montagne, engendre un environnement socio-économique complexe qui influence la structure de son offre en matière de tourisme d'aventure. Depuis la perestroïka, trois générations d'entreprises se sont succédé. Elles ont diversifié l'offre touristique en proposant des voyages plus culturels et moins collectifs, mais restent spécialisées sur un type de produit, une région ou un moyen de déplacement. Cette logique de spécialisation différencie fortement l'espace de concurrence kirghiz de son équivalent français puisque les agences françaises 
diversifient au maximum leurs offres afin de répondre à toutes les attentes que pourraient avoir les différentes composantes de leur clientèle.

De plus, les agences françaises traitent en priorité avec des réceptifs fondés par des expatriés, qui ont accumulé différentes formes de capitaux pour pouvoir s'implanter : un capital international constitué de formations supérieures et d'expériences professionnelles, mais aussi des ressources sociales alimentées par des stratégies matrimoniales. Ainsi, le partage d'une même culture, d'une même langue, la connaissance implicite des attentes de la clientèle française en matière d'encadrement, de confort, de qualité des prestations, de relations privilégiées avec les populations locales favorisent l'établissement de relations de confiance, rétablissent une proximité symbolique nécessaire pour ces échanges commerciaux à distance.

Ces conditions de choix des correspondants nous conduisent à rejeter l'hypothèse d'une homologie entre les deux espaces puisque leur relation n'est pas directe : les agences dominantes dans un espace ne peuvent se contenter de traiter avec celles qui dominent l'autre. Pour les activités très techniques comme l'alpinisme, les agences françaises font appel à une des premières agences kirghizes créées à l'époque soviétique. Pour le reste, elles ont des correspondants de taille très différente, en fonction de leur spécialité, la priorité allant plutôt aux Français expatriés. D'un autre côté, les agences kirghizes ont de nombreux partenaires français en fonction de leur positionnement initial, de leurs contrats d'exclusivité, de leurs expériences. Par exemple, l'agence Bleu est la plus reconnue parmi les expatriés français, mais elle ne travaille pas avec Terres d'Aventure. Ces deux espaces de production ont des logiques de fonctionnement proches, mais les partenariats entre agences sont croisés et multiples.

En définitive, il apparaît que les réceptifs fondés par les expatriés se sont adaptés à des conditions difficiles pour se faire une place dans un espace de production existant. Ils ont bénéficié néanmoins d'un avantage concurrentiel primordial pour traiter avec les agences françaises : la proximité culturelle. Ils participent également à transformer l'offre kirghize en important des modèles de fonctionnement et des exigences différentes de ceux qui sont développés localement.

\section{Références}

ANDREFF, Wladimir (2007) Économie de la transition. La transformation des économies planifiées en économies de marché, Rosny-sous-Bois : Bréal. $494 \mathrm{p}$.

Anonyme (2005) fondateur et directeur de l'agence Bleu, entretien réalisé à Bourg-Saint-Maurice, France, le 15 avril.

Anonyme (2006a) fondateur et directeur de l'agence Bleu, entretien réalisé à Bishkek, Kirghizstan, le 21 septembre.

Anonyme (2006b) fondateurs de l'agence Jaune, entretien réalisé à Bishkek, Kirghizstan, le 30 septembre.

BARDIN, Laurence (1993) L'analyse de contenu, Paris : Presses Universitaires de France. 291 p.

BOLTANSKI, Luc (1976) «L'encombrement et la maîtrise des “biens sans maître”", Actes de la Recherche en Sciences Sociales, vol. 2, n 1, p. 102-109.

BOTTI, Laurent; Nicolas PEYPOCH et Bernardin SOLONANDRASANA (2008) Ingénierie du tourisme. Concepts, méthodes, applications, Bruxelles : De Boeck. 168 p.
BOURDIEU, Pierre (1972) «Les stratégies matrimoniales dans le système de reproduction ", Annales Économie, sociétés, civilisations, vol. 27, n 4-5, p. $1105-1127$.

BOURDIEU, Pierre (1979) La distinction. Critique sociale du jugement, Paris : Les Éditions de Minuit, Coll. «Le sens commun». 672 p.

BOURDIEU, Pierre (1992) Les règles de l'art, genèse et structure du champ littéraire, Paris : Seuil. 480 p.

BRIAND, Y. (2003) directeur de production chez Club Aventure, entretien réalisé à Marseille, le 18 mars.

CACCOMO, Jean-Louis et Bernardin SOLONANDRASANA (2001) «De l'idée au produit. L'innovation touristique, un effort difficile à mesurer ", Revue Espaces, $\mathrm{n}^{\circ} 186$, p. 37-40.

CACCOMO, Jean-Louis et Bernardin SOLONANDRASANA (2006) L'innovation dans l'industrie touristique. Enjeux et stratégies, Paris : L’Harmattan, Coll. «Tourismes et sociétés». 180 p.

CHAN, Su Han; George W. GAU et Ko WANG (1995) «Stock Market Reaction to Capital Investment Decisions: Evidence from Business Relocations », Journal of Financial and Quantitative Analysis, vol. 30, n 1 , p. $81-100$.

CHANDLER, Gaylen N. et Erik JANSEN (1992) «The Founder's Self-assessed Competence and Venture Performance», Journal of Business Venturing, vol. 7, no 3, p. 223-235.

DE GRANDPRÉ, François (2007) «Attraits, attractions et produits touristiques : trois concepts distincts dans le contexte d'un développement touristique régional», Téoros, vol. 26, n 2, p. 12-18.

DÉSERT, Myriam (2006) «Le débat russe sur l'informel», Questions de recherche, $\mathrm{n}^{\circ} 17$, p. 2-53.

DOMPIER, R. (2003) cofondateur, codirecteur et responsable de communication chez Tirawa, entretien réalisé à Chambéry, le 8 avril.

DURAND, Cédric (2005) «Les privatisations en Russie et la naissance d'un capitalisme oligarchique», Recherches internationales, vol. 74, $\mathrm{n}^{\circ} 4$, p. 33-50.

FAFCHAMPS, Marcel (2002) Social capital and development, Working paper, Oxford : University of Oxford. 13 p.

GÉRAUD, Marie-Odile (2002) «Esthétiques de l'authenticité. Tourisme et touristes chez les Hmong de Guyane française », Ethnologie Française, vol. 32, no 3, p. 447-459.

GLAESER, Edward L. (2001) «The formation of the social capital», Canadian Journal of Policy Research, vol. 2, n 1, p. 34-40.

HALLÉ, Julie (2008) «L'état de l'organisation du tourisme sportif dans les pays d'Asie centrale (Kazakhstan, Kirghizstan, Ouzbékistan, Tadjikistan). Acteurs et structures dans un modèle en transition ». Thèse en sciences et techniques des activités physiques et sportives non publiée, Grenoble : Université Joseph Fourier. 634 p.

JOHANSON, Jan et Jan-Erik VAHLNE (1990) «The mechanism of internationalization ", International Marketing Review, vol. 7, n 4, p. 11-24.

JOUTY, Sylvain et Hubert ODIER (1999) Dictionnaire de la montagne, Paris : Arthaud, $1024 \mathrm{p}$.

LE BRETON, David (1996) L'aventure ou la passion des détours, Paris : Autrement, $210 \mathrm{p}$.

LEHTINEN, Uolevi et Heli PENTTINEN (1999) «Definition of the internationalization of the firm », DANS LEHTINEN, Uolevi et Hannu SERISTÖ (sous la direction de), Perspectives on internationalization, p. 3-19. Helsinki : Helsinki School of Economics and Business Administration.

LERMAN, Zvi (2009) «Land reform, farm structure, and agricultural performance in CIS countries ", China Economic Review, vol. 20, n 2, p. 316-326. 
MILL, D. (2003) directeur de production chez Allibert, entretien réalisé à Chapareillan, le 7 avril.

PABION-MOURIÈS, Johanne (2011) «L'écotourisme comme facteur d'ethnicisation de la société kirghize post-soviétique», Grotius International, $<$ http://www.grotius.fr/lecotourisme-comme-facteur-dethnicisation-dela-societe-kirghize-post-sovietique/>, consulté le 20 janvier 2012.

PABION-MOURIÈS, Johanne (2012) «L'écoutourisme des jailoo », EspacesTemps.net, <http://espacestemps.net/document9524.html>, consulté le 10 septembre 2012.

PASSAVANT, Eric (1999) «Comment devient-on leader européen du tourisme? Analyse stratégique de l'agence Terres d'Aventure», Actes du Troisième congrès de la Société Française de Management du Sport (Ronchin : 9-11 décembre), p. 143-152.

PASSAVANT, Eric (2002) «Aventure sensible et aventure pittoresque : La dynamique de l'espace de production du tourisme d'aventure », Alinéa Sciences Sociales et Humaines, $\mathrm{n}^{\circ} 3$, p. 88-104.

PÉTRIC, Boris-Mathieu (2002) Pouvoir, don et réseaux en Ouzbékistan postsoviétique, Vendôme : Presses Universitaires France. 297 p.

PÉTRIC, Boris-Mathieu (2006) «Le Kirghizstan, royaume des ONG», OutreTerre, $\mathrm{n}^{\circ} 16$, p. 287-301.

PÉTRIC, Boris-Mathieu (2007) «Le Kirghizstan : lieu d'expérimentation pour mesurer les nouvelles normes de la mondialisation politique», Cahiers du GEMDEV, nº 31, p. 34-51.

PONS, Gérard et Grégoire DE LA FOUCHARDIÈRE (2003) «Spécialisation thématique des agences. Des agences fortes en thème », Revue Espaces, $n^{\circ} 203$, p. 27-29.
PORTER, Michael (1982) Choix stratégique et concurrence, Paris : Economica. $426 \mathrm{p}$.

RÉAU, Bertrand (2011) Les Français et les vacances. Sociologie des pratiques et offres de loisir, Paris : CNRS. 235 p.

REUBER, A. Rebecca et Eileen FISCHER (1997) «The influence of the management team's international experience on the internationalization behaviors of SMEs », Journal of International Business Studies, vol. 28, $\mathrm{n}^{\circ} 4$, p. 807-825.

TERAUDS, Kris (2010) Transition agraire : le cas divergent de l'Ouzbékistan et $d u$ Kirghizstan, Genève : Institut des Hautes Études Internationales et du Développement. 25 p.

THOREZ, Julien (2006) «Flux et dynamiques spatiales en Asie centrale. Géographie de la transformation post-soviétique», ESO, n 24, p. 65-69.

TINARD, Yves (1992) «Le tourisme d'aventure, un concept évolutif», Les cahiers Espaces, n 29, p. 8-19.

TRUCHET, Stéphanie; Francis AUBERT et Jean-Marc CALLOIS (2008) "Capital social et entrepreneurs touristiques ruraux», Présentation orale, $2^{e}$ journée de recherche en sciences sociales non publiée, INRA SFER CIRAD (Lille : 11-12 décembre). $23 \mathrm{p}$.

URBAIN, Jean-Didier (1991) L'idiot du voyage : histoires de touristes, Paris : Payot. 271 p.

WAGNER, Anne-Catherine (1998) Les nouvelles élites de la mondialisation. Une immigration dorée en France, Paris : Presses Universitaires de France. $240 \mathrm{p}$.

WAGNER, Anne-Catherine (2007) Les classes sociales dans la mondialisation, Paris : La Découverte. 128 p.

\section{APPROCHES MÉTHODOLOGIQUES EN TOURISME • APPEL À TEXTES}

Qu'elle soit qualitative ou quantitative, la recherche implique le recours à des techniques de collecte de données et d'analyses qui ont fait leurs preuves. II arrive cependant que l'expérience touristique, par sa nature particulière (l'étude de phénomènes ou pratiques où certains acteurs sont en mode opérationnels alors que les prestataires sont en mode repos), nécessite une approche particulière. Considérant la nature multidisciplinaire du tourisme, et l'évolution rapides des expériences offertes, les chercheur(e)s sont ainsi confronté(e)s à des défis particuliers.

II y a un besoin réel de trouver des solutions adaptées aux problèmes engendrés par la recherche en tourisme et de développer des outils adaptés aux situations particulières du domaine. II est tout aussi important de jeter un regard critique sur les méthodologies employées jusqu'à présent en recherche touristique.

Téoros est à la recherche de textes consacrés à la méthodologie de recherche en tourisme, soit à propos de nouvelles approches, soit à propos de nouvelles façons de recourir aux méthodes de recherches existantes. Seront considérés les articles développés autour de discussions conceptuelles ou encore sur les applications de diverses méthodes. En plus de ces aspects, votre article peut porter sur:

- de nouvelles méthodes de recherche en tourisme selon les disciplines concernées par le phénomène;

- de nouvelles façons d'employer des méthodes de recherche conventionnelles en tourisme;
- des façons créatives de combiner les méthodes qualitative et quantitative;

- de nouvelles approches pour l'étude des questions contemporaines qui impliquent l'effort de communications entre les disciplines (comme, par exemple, la nature interdisciplinaire croissante des études en tourisme et le développement accéléré des études virtuelles).

Les auteur(e)s doivent faire parvenir un manuscrit rédigé préférablement en français présenté selon les règles de la revue, disponibles à l'url: http://teoros.revues.org/168. Les textes soumis, en format Word, doivent compter environ 7000 à 7500 mots et doivent comprendre un objectif (question) de recherche clairement énoncé; un descriptif de la méthodologie de recherche employée, et un volet théorique. Une étude de cas peut s'ajouter à ces éléments.

Chaque article doit inclure les nom et prénom de tous les auteurs, leur titre principal et leur affiliation (une seule), leur adresse électronique (courriel) et postale, un résumé de 150 à 200 mots maximum en français ainsi qu'une une liste des mots clés (maximum de 5). II n'y a pas de date limite pour soumettre un texte sur les approches méthodologiques: Téoros les reçoit en tout temps. Les propositions de textes doivent être adressées à la revue: teoros@ uqam.ca. Veuillez inscrire "Méthodologie» dans la ligne de sujet.

Au plaisir de vous lire dans nos pages! 